\title{
Integrative genomic analyses on GLI2: Mechanism of Hedgehog priming through basal GLI2 expression, and interaction map of stem cell signaling network with P53
}

\author{
YURIKO KATOH $^{1}$ and MASARU KATOH ${ }^{2}$ \\ ${ }^{1}$ M\&M Medical BioInformatics, Hongo 113-0033; ${ }^{2}$ Genetics and Cell Biology Section, \\ National Cancer Center Research Institute, Tokyo 104-0045, Japan \\ Received June 17, 2008; Accepted July 18, 2008 \\ DOI: 10.3892/ijo_00000076
}

\begin{abstract}
Hedgehog-binding to Patched family receptors results in Smoothened-mediated activation of MAP3K10 (MST) and inactivation of SUFU. MAP3K10-induced DYRK2 phosphorylation combined with SUFU inhibition results in the stabilization and nuclear accumulation of GLI2 for transcriptional activation of $G L I 1, C C N D 1, C C N D 2, F O X A 2$, FOXC2, FOXP3, FOXQ1, RUNX2, and JAG2. Here, integrative genomic analyses on GLI2 orthologs were carried out. Rat Gli2 complete coding sequence was determined by assembling nucleotide sequences of exons 1,2, and 5'truncated rat Gli2 RefSeq (NM_001107169.1). GLI2 orthologs were more related to GLI3 orthologs than to GLI1 orthologs lacking the N-terminal repressor domain. BTRCP1 (FBXW1)-binding DSYxxxS motif was conserved in GLI2 and GLI3 orthologs, while ßTRCP2 (FBXW11)-binding DSGxxxxxxxxxS motif in GLI2 and GLI1 orthologs. Human GLI2 mRNA was expressed in ES cells, NT2 cells, fetal lung, fetal heart, regenerating liver, gastric cancer, and other tumors. Mouse Gli2 mRNA was expressed in unfertilized egg, ES cells, and EG cells. Tandem RRRCWWGYYY motifs for P53, P63 or P73, and also four conserved bHLHbinding sites were identified within GLI2 proximal promoter region. Interaction map of P53 and stem cell signaling network were then constructed. P53-induced NOTCH1 upregulation leads to HES1, HES5, HEY1, HEY2 or HEYL upregulation for the repression of tissue specific bHLH transcriptional activators. DYRK2 functions as a positive regulator of P53-mediated apoptosis, and also as a negative regulator of the Hedgehog signaling cascade. GLI2 expression is regulated based on the balance of P53, Notch, and TGF- $ß$ signaling, and Hedgehog signaling activation
\end{abstract}

Correspondence to: Dr Masaru Katoh, Genetics and Cell Biology Section, National Cancer Center Research Institute, 5-1-1 Tsukiji, Chuo-ku, Tokyo 104-0045, Japan

E-mail: mkatoh-kkr@umin.ac.jp

Key words: Hedgehog, Smoothened, P53, P63, P73, MDM2, MDM4, MDMX, EGFR, AKT, HIPK2, GSK-3ß, protein kinase A, Notch, WNT results in cell survival and proliferation due to transcriptional activation of Hedgehog-target genes, and also partly due to perturbation of P53-mediated transcriptional regulation.

\section{Introduction}

Sonic Hedgehog (SHH), Indian Hedgehog (IHH), and Desert Hedgehog (DHH) are Hedgehog family members, regulating embryogenesis, and adult tissue homeostasis, and carcinogenesis (1-5). Hedgehog signaling is activated in various human tumors, such as basal cell carcinoma, melanoma, small cell lung cancer, esophageal cancer, gastric cancer, pancreatic cancer, and malignant lymphoma (6-14).

Hedgehog precursors are processed to cut off the C-, and $\mathrm{N}$-terminal regions for cholesteroylation and palmitoylation, respectively $(15,16)$. Mature Hedgehog proteins are then transported to the cell surface for DISP1-dependent packaging into lipoprotein particles, or for lipophilic tail-mediated multimerization $(16,17)$. PTCH1 and PTCH2 are Hedgehog receptors, while CDON, BOC, and GAS1 are Hedgehog coreceptors $(6,18-20)$. Hedgehog-binding to Patched family receptors results in Smoothened signaling activation due to the Hedgehog-mediated internalization of Patched family receptors suppressing Smoothened function $(21,22)$. Hedgehoginduced Smoothened activation leads to MAP3K10 (MST) activation and SUFU inactivation $(23,24)$.

GLI1, GLI2, and GLI3 constitute the GLI family of transcription factors $(25,26)$. GLI1 consists of zinc finger domains, and C-terminal activator domain. On the other hand, GLI2 and GLI3 consist of N-terminal repressor domain, zinc finger domains, and C-terminal activator domain. In the absence of Hedgehog-induced Smoothened activation, GLI1 is transcriptionally repressed, GLI2 is phosphorylated for the BTRCP2 (FBXW11)-mediated degradation, and GLI3 is phosphorylated for the BTRCP1 (FBXW1)-mediated processing into repressor lacking the $\mathrm{C}$-terminal activator domain (26-28). In the presence of Hedgehog-induced Smoothened activation, MAP3K10 activation and SUFU inactivation leads to the stabilization and nuclear translocation of GLI2, which results in transcriptional activation of target genes, including GLII, CCND1, CCND2, FOXA2, FOXC2, FOXP3, FOXQ1, RUNX2, and JAG2 (29,30). GLI1 upregulation augments the Hedgehog signaling cascades through positive-feedback mechanism, 
while Cyclin D upregulation promotes cellular proliferation. Therefore, GLI2 is the key molecule implicated in the initial activation of the Hedgehog signaling cascades.

Because transcriptional mechanism of GLI2 remains almost unclear, we carried out integrative genomic analyses on GLI2 orthologs. Complete coding sequence of rat Gli2 was determined for the comparative proteomic analyses. Complete tandem P53-binding sites and four bHLH-binding sites were next identified within proximal promoter region of human GLI2 promoter. Then, interaction map of GLI2 and P53 were constructed with the emphasis on the stem cell signaling network.

\section{Materials and methods}

Comparative genomic analyses. Rat genome sequences homologous to human GLI2 and mouse Gli2 were searched for by using the BLAST programs as previously described $(31,32)$. Conserved transcription factor-binding sites within promoter regions were then searched for based on the Match program, and manual curation as previously described $(33,34)$.

Comparative proteomic analyses. CLUSTALW program was used for phylogenetic analysis on human and rodent GLI family members. Amino-acid sequences of human GLI1 (NP_005260.1), GLI2 (NP_005261.2), GLI3 (NP_000159.3), mouse Gli1 (NP_034426.2), Gli2 (NP_001074594.1), Gli3 (NP_032156.2), and rat Gli2 (Fig. 1) were used for the phylogenetic analysis.

In silico expression analyses. Expressed sequence tags (ESTs) derived from human GLI2 and mouse Gli2 were searched for using the BLAST programs as previously described $(35,36)$. Human GLI2 RefSeq (NM_005270.3) and mouse Gli2 RefSeq (NM_001081125.1) were used as query sequences for the BLAST programs.

\section{Results}

Complete coding sequence of rat Gli2. Preliminary phylogenetic analyses on the mammalian GLI family members revealed that rat Gli2 protein RefSeq (NP_001100639.1) deduced from rat Gli2 nucleotide RefSeq (NM_001107169.1) was $\mathrm{N}$-terminally truncated. Comparative genomic analyses next revealed that the 5'-truncation of NM_001107169.1 RefSeq was due to the lack of nucleotide sequences corresponding to exons 1 and 2 of rat Gli2 gene. Therefore, we decided to determine the complete coding sequence of rat Gli2 transcript in this study.

BLAST programs revealed that missing exons 1 and 2 of the rat Gli2 gene were located within rat genome sequence AC120834.4. Exon 1 corresponded to the complementary sequence for nucleotide position 200441-200128 of AC120834.4, and exon 2 to the complementary sequence for nucleotide position 151596-151421. Complete coding sequence of rat Gli2 was then determined by assembling nucleotide sequences of exons 1, 2, and 5'-truncated RefSeq NM_001107169.1. Because nucleotide position 346-4980 was the coding region of rat Gli2 complete coding sequence, rat Gli2 gene was found to encode 1544 amino-acid Gli2 protein (Fig. 1A).

Comparative proteomics on mammalian GLI family members. Refined phylogenetic analysis on the GLI family members using the full-length rat Gli2 protein revealed that GLI2 orthologs were more related to GLI3 orthologs than to GLI1 orthologs (Fig. 1B). Alignment of mammalian GLI family members next revealed that GLI1 orthologs were shorter than GLI2 and GLI3 orthologs due to the lack of the N-terminal repressor domain.

Bhatia et al reported that DSGxxxxxxxxxS motif in GLI2 orthologs is the BTRCP2-binding site to induce degradation (27), and Tempé et al reported that DSYxxxS motif in Gli3 is the BTRCP1-binding site to induce proteolysis (28). We found that the DSGxxxxxxxxxS motif was conserved in GLI2 and GLI1 orthologs, and also that the DSYxxxS motif was conserved in GLI2 and GLI3 orthologs (Fig. 1C and D).

Expression profile of GLI2 orthologs. Human GLI2 mRNA was expressed in ES cells, NT2 cells, fetal lung, fetal heart, regenerating liver, gastric cancer, ovarian cancer, prostate cancer, acute myelogenous leukemia, neuroblastoma, and glioblastoma. Mouse Gli2 mRNA was expressed in unfertilized egg, ES cells, and EG cells.

Comparative genomics on mammalian GLI2 orthologs. Because the nucleotide sequence of proximal promoter region of rat Gli2 gene was homologous to 5'-region of human GLI2 exon 1 a rather than that of exon $1 \mathrm{~b}$, comparative genomic analyses were focused on the 5'-region of human GLI2 exon 1a in this study.

Transcription factor-binding sites within the 5'-region of human GLI2 exon 1a were then searched for. Although it was previously reported that TGFß induces Smad3-mediated GLI2 upregulation in human fibroblasts, keratinocytes, and various cancer cell lines (37), Smad3-binding site within the 5'-region of human GLI2 exon 1a was not successfully identified due to the too redundant sequences of Smad-3-binding sites. Instead, basic helix-loop-helix (bHLH)-binding sites, and tandem RRRCWWGYYY motifs without any space for P53, P63 or P73 transcription factors were identified within the $1.5-\mathrm{kb}$ region just upstream of the transcriptional start site of human GLI2 exon 1a (Fig. 2A). Four basic helix-loop-helix (bHLH)-binding sites within human GLI2 promoter were conserved in the mouse Gli2 promoter.

Tandem P53-binding motifs within human GLI2 promoter were not conserved in rodents due to significant regional divergence, and not in chimpanzee due to $\mathrm{C}>\mathrm{T}$ nucleotide substitution at the consensus $\mathrm{C}$ residue of the first P53-binding motif. Because the tandem P53-binding motifs within the GLI2 promoter were destroyed in chimpanzee GLI2 promoter due to single nucleotide substitution, we next searched for a single nucleotide polymorphism (SNP) at that position. However, we could not detect any SNP at the P53-binding site within GLI2 promoter at least in AC017033.5 and AC016764.8 BAC sequences and personal genome sequences of Dr Venter and Dr Watson.

Together these facts indicate that four bHLH-binding sites within GLI2 promoter were evolutionarily conserved, and 

MNPHMEHYLRSVHSSPTLSMISAARGLSPADVAHEHLKERGLFSLAAPGTNPSDYYHQMTLMASHPTPYGDILMQSGGAASAPHLHDYLNPVDASRFSSPRVTPRLSRKRALSISPLSDA SLDLQRMIRTSPNSLVAYINNSRSSSAASGSYGHLSAGALSPAFTFPHPINPVAYQQILSQQRGLGSAFGHTPPLIQPSPTFLAQQPMTLTSITVPTQLGSNSSNCISDANQNKQNSESA VSTWF GEKPHKCTFEGCSKAYSRLENLKTHLRSHTGEKPYVCEHEGCNKAFSNASDRAKHQNRTHSNEKPYICKIPGCTKRYTDPSSLRKHVKTVHGPDAHVTKKQRNDVHLRAPLLKENGDNEA KLKSLKDSCSWAGPAPHTRNTKLPPLPVWGSVL ENFENTTGGCGPAGLLPNORLPELTEVTMLSOIOERRDSSTSTMSSAYTVSRRSSGISPYFSSRRSSEASPIGGIRPHMASSADSYDP ISTDASRRSSEASOCSGGG GLLNLTPAOOYSLRAKYAAATGGPPPTPLPGLDRVSLRTRLALDAPERALPGACPOPLGPRRGSDGPTYNHGHGYVGAAPALPHEGPNGSTRRASDPVR RPDPLVLPRVQRFHSIHNMNPGPLPPCTDRRGLHLOSHPSVDGSLTRHAYSPRPPSINENVVMEAVAAGVEGAGLESDLGLVEDDLVLPDDVVOYIKAHTGGALDDSTROVYPTEGTGFP ENSKLPSPGLQGHRRLAAADSNMSPSAPGLGGCQLSYSPSSSLNKSNMPVQWNEVSSGTVDALPTQVKPPSFPQSNLVQQKPAFAHYPGYSPQALQSGSGGLDSTQPHLQLRGAPSASRG SYTQQPRLPATGSQCLGVTAAMSPQANYSQTHHQLSPNVVSGSLNOFAPSCSNMAAKPSHLGLPQQMEVVPNATIISGHQRELGIASSSLAAVSQPHPVLGYPQQDGYQVSGLPSSHQP SFMESQQNTGFGLMQPRPPLEPNTASRHRGVRSGQQQQQLYARTAGQAVVTSANQETAEAMPKGTAGTVVSLTPQPSQDTGRSQDQNTLYYYGQIHMYEQNGGCPAMQPQPPQPQGCSDN IQPEPLPSPGVNQVSSTVDSQLLEPPQIDFDAIMDDGDHSSLFSGALSPSLLHNLSQNSSRLTTPRNSLTLPSTPVGISNMAVGDMSSMLTSLAEENKFLNMMT

B

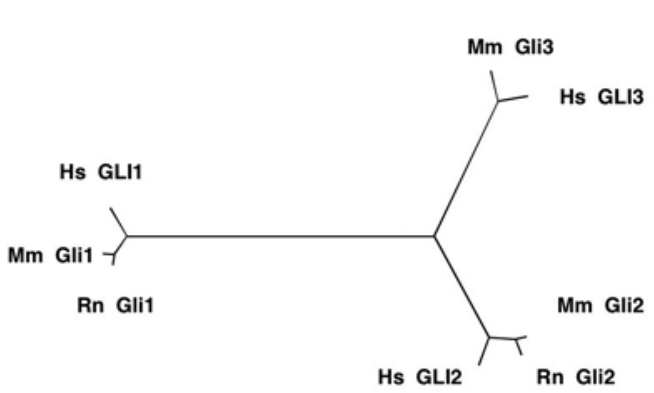

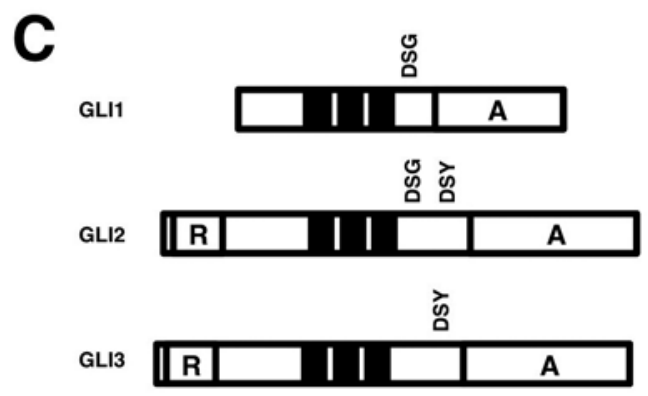

DSGXxxxxxxxxS

D

Figure 1. Comparative proteomics on the GLI family. (A), Amino-acid sequence of rat Gli2. (B), Phylogenetic tree of human and rodent GLI family members. (C), Domain architecture of GLI orthologs. R, repressor domain; A, activator domain; DSG, DSGxxxxxxxxS motif; DSY, DSYxxxS motif; closed boxes, zinc finger domains. (D), Partial alignment of GLI family members around the DSGxxxxxxxxxS and DSYxxxS motifs.

that the tandem P53-binding motifs within the GLI2 promoter were human specific.

Interaction map of P53 with stem cell signaling network. Hedgehog, BMP/TGFß/Nodal/Activin, EGF/FGF, Notch, and WNT signaling cascades constitute stem cell signaling network (3,38-41). Because both P53 and stem cell signaling network are implicated in carcinogenesis, we next constructed the interaction map of P53 with stem cell signaling network.

P53-induced Notch1 upregulation $(42,43)$ leads to upregulation of Hes/Hey family members to repress tissue specific bHLH transcriptional activators $(36,44-46)$. bHLH and P53 are predicted to regulate GLI2 transcription (Fig. 2A), and TGF- $\beta$ signaling to Smad3 is also implicated in GLI2 upregulation as mentioned above (37). Together these facts indicate that GLI2 expression is regulated based on the balance of P53, Notch, and TGF- 3 signaling (Fig. 2B).

We cloned and characterized MAP3K10 (MST) as a novel serine/threonine kinase derived from MKN28 gastric cancer cells in 1995 (47), and Taipale's group identified MAP3K10 as a Hedgehog signaling component in 2008 (23). MAP3K10 is reported to directly phosphorylate DYRK2 for its functional inhibition (23). DYRK2 functions as a negative regulator of the Hedgehog signaling cascade (23), but also as a positive regulator of P53-mediated apoptosis (24). GLI2-mediated Hedgehog signaling activation results in cell survival and proliferation due to transcriptional activation of Hedgehogtarget genes, and also partly due to perturbation of P53mediated transcriptional regulation (Fig. 2B).

\section{Discussion}

Complete coding sequence of rat Gli2 was determined in this study (Fig. 1A). GLI2 and GLI3 orthologs shared the common domain architecture, consisting of $\mathrm{N}$-terminal repressor domain, zinc finger domains, BTRCP1-binding DSYxxxS motif, and C-terminal activator domain (Fig. 1C).

Although GLI2 orthologs were more related to GLI3 orthologs than to GLI1 orthologs, BTRCP2-binding DSGxxxxx xxxxS motif was conserved in GLI2 and GLI1 orthologs, but not in GLI3 orthologs (Fig. 1C). DYRK2, PKA and GSK3ß phosphorylate GLI2 in the absence of Hedgehog signaling $(23,48)$, and phosphorylation of the DSGxxxxxxxxxS motif in GLI2 leads to BTRCP2-mediated ubiquitination and subsequent proteasome-dependent degradation (27). In the absence of Hedgehog signaling, GLII mRNA is transcriptionally repressed, and GLI1 protein might also be degraded due to the same mechanism as GLI2 degradation. 
A

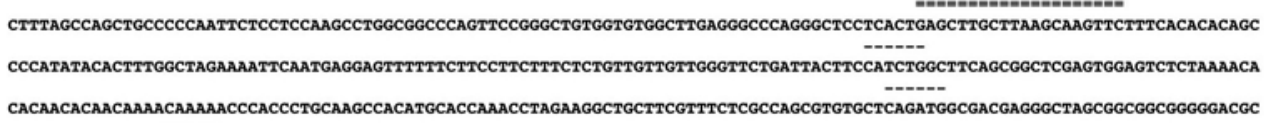
CACAACACAACAAAACAAAAACCCACCCTGCAAGCCACATGCACCAAACCTAGAAGGCTGCTTCGTTTCTCGCCAGCGTGTGCTCAGATGGCGACGAGGGCTAGCGGCGGCGGGGGACGC CTCAGGACCCCCGGCACCTGCGCTCGCTGCCCGCGGGAAGAGGGTCCGCGTGGACCCAGCCCCCGCGTCCCTTCTCTGGCGTCCCCGGCTTCCGCGCGGGCGTCCAGAGAAGCGGGCGCC TGAGTTAGCGACACCCCCAACCCCCCCCCCCACACCTAGTCGTCTAAAAAAAGTGTCGGAGATTGGCGCGTCCTTTGGTTCCTTTCTCGAACTTTCCTTGTAGGTGCGTTTTCTTTCCTT GGTGCTGGGTGGGGAGAGTCCCTGTGCTCCCCCTTTCCCCTCCCCCCGCCCCGGCGGCGTTCGGGTCCCCCTGCGTCCCCCGGCAGGGAGCGGGCGGGCTGGCTGGCGGGTTCTTGGGGC CCGGGTGTGCCCGCACCGTGCGCGCGGGGGCGCTGCGCAGTCCGGCGGCGCTGATGGATTGCAGAAGTGCCGGCGCTTGCCAGCCGAGGCAGCACGGCTCCGCGGACTTTTTTTCAAACT CCCATCAATGAGACTTCGAGGAGGAGCGGGCGGCGGCGGCGGCTGCGACTGCGAACGCGGAGGAAGGCCAGGAGCCGCAGGAGGAGCCGGAGGAAAGAGCTTGGGCCGCGCGGCGCGCCG CAGCCTCGGGGAGCCGCCTGCTCGCCGGCGGTAGGGGCTGCGCGGCGCCCGCCCGCCTCTCGGTCCCCTCTCTTGCCTGGCCCGCCCCGCCCCGGCTGGCTGGAGCCCCGGCACAAGGCA GCCAGCCGAGGGTCGCCGCGCCAGCCAAGGTGGGATGGGGGCCCACAGCCACCGCCCGGCGCCCGAGAGGCCACCTGCGTGCTAGAGGCAAACTTTTGTCTCTCTCGGTAAAGTTGCATT

B

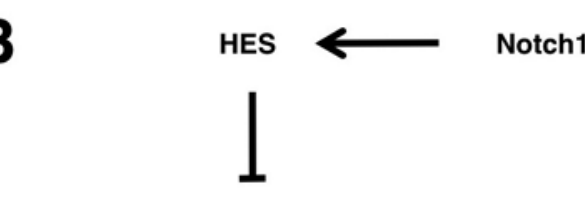

?

$\rightarrow$ bHLH
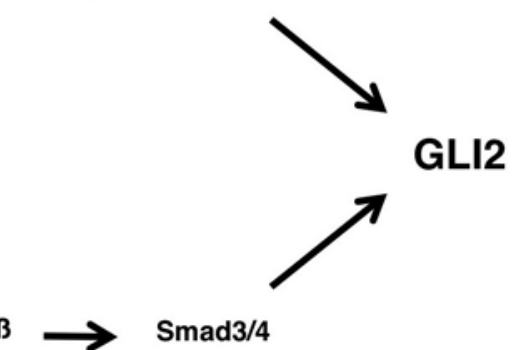

Notch1
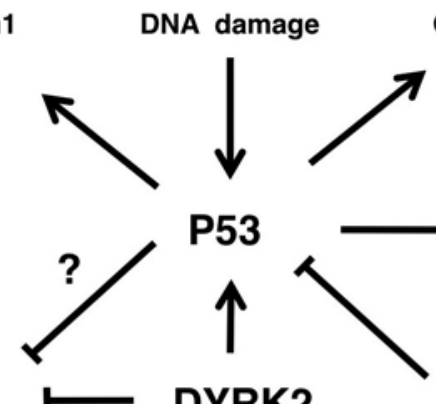

P53
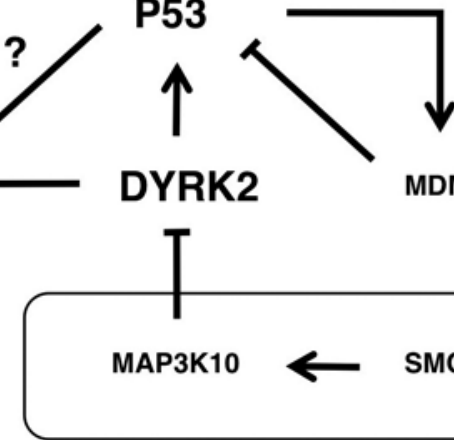

Growth arrest or Apoptosis

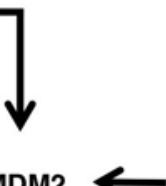

FOXO
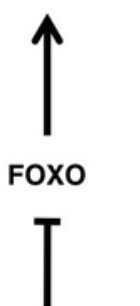

AKT

RTK

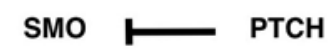

$\mathrm{HH}$

Figure 2. GLI2 and P53. (A), Proximal promoter region of human GLI2. Tandem P53-binding sites (double overline), conserved bHLH-binding sites (overline), and exonic region (open box) are indicated. (B), Interaction map of P53 and stem cell signaling network. DYRK2 is located at the crossroads of Hedgehog and P53 signaling cascades.

We identified tandem P53-binding motifs within the human GLI2 promoter (Fig. 2A). GLI2 was not listed on the 542 target genes or loci of P53 identified by using ChIP-on-PET approach (42), nor on the 1546 targets of P53 identified by using ChIP-on-chip approach (49). P53, P63, and P73 bind to the tandem RRRCWWGYYY motifs to activate or repress their target genes. Although transcriptional regulation of GLI2 by P53, P63, and P73 should be further investigated, this is the first report on the tandem P53-binding motifs within the proximal promoter region of human GLI2 gene.

DYRK2 phosphorylates P53 to enhance apoptotic response to DNA damage (50), while DYRK2 phosphorylates GLI2 and NFATc to prime subsequent phosphorylation by GSK3ß for degradation and inhibition of nuclear translocation, respectively $(23,51)$. Hedgehog signaling activation leads to DYRK2 inhibition in the cytoplasm, which might lead to inhibition of DYRK2-mediated P53 activity in the nucleus. Because DYRK2, HIPK2, PKC $\delta$ and P38 are implicated in P53 activation (50), Hedgehog signals are predicted to partially inhibit P53 activity in cancer cells.

It was expected that $D Y R K 2$ gene might be deleted, mutated or silenced in some cancer as a tumor suppressor gene; however, DYRK2 gene is amplified and overexpressed in lung cancer, esophageal cancer, and gastric cancer $(52,53)$.
The mechanism how DYRK2 overexpression leads to survival or proliferation of tumor cells remains to be elucidated.

MDM2 and MDM4 (MDMX) are negative regulators of P53. MDM2 and MDM4 share the common domain architecture consisting of P53-binding domain, acidic domain, zinc finger domain, and RING finger domain (54-57). MDM2 gene, located at human chromosome $12 \mathrm{q} 15$, is amplified and overexpressed in osteosarcoma, soft tissues sarcoma, gastric cancer, lung cancer, and esophageal cancer $(52,55,58)$. MDM4 gene, located at human chromosome 1q32.1, is amplified and overexpressed in malignant glioma, breast cancer, and retinoblastoma (59-61). MDM2 and MDM4 function as oncogenes, because MDM2 and MDM4 proteins protect tumor cells from apopotosis or growth arrest due to their inhibitory effect on P53 tumor suppressor.

Receptor tyrosine kinase (RTK)-mediated or RTK-induced PI3K-AKT signaling activation leads MDM2 phosphorylation at Ser 166 and Ser 186 to inhibit P53 activity (62). Because the activation of Hedgehog signaling and RTK signaling synergistically inhibits P53 function (Fig. 2B), monitoring of P53, Hedgehog, and AKT activities in primary tumors could be utilized for the prediction of effectiveness of DNA-damaging agent or irradiation to induce P53-mediated apoptosis in cancer cells. In addition, combination chemotherapy using 
Hedgehog inhibitor and RTK inhibitor could promote antitumor effects of chemotherapy and/or irradiation.

\section{References}

1. Marigo V, Roberts DJ, Lee SM, et al: Cloning, expression, and chromosomal location of $\mathrm{SHH}$ and $\mathrm{IHH}$. Genomics 28: 44-51, 1995.

2. Beachy PA, Karhadkar SS and Berman DM: Tissue repair and stem cell renewal in carcinogenesis. Nature 432: 324-331, 2004.

3. Van den Brink GR, Bleuming SA, Hardwick JC, et al: Indian Hedgehog is an antagonist of Wnt signaling in colonic epithelial cell differentiation. Nat Genet 36: 277-282, 2004.

4. Hooper JF and Scott MP: Communicating with Hedgehogs. Nature Rev Mol Cell Biol 6: 306-317, 2005.

5. Katoh Y and Katoh M: Hedgehog signaling pathway and gastrointestinal stem cell signaling network. Int J Mol Med 18: 1019-1023, 2006

6. Johnson RL, Rothman AL, Xie J, et al: Human homolog of patched, a candidate gene for the basal cell nevus syndrome. Science 272: 1668-1671, 1996.

7. Stone DM, Hynes M, Armanini M, et al: The tumour-suppressor gene Patched encodes a candidate receptor for Sonic hedgehog. Nature 384: 129-134, 1996.

8. Dahmane N, Lee J, Robins P, Heller P and Ruiz i Altaba A: Activation of the transcription factor Glil and the Sonic hedgehog signalling pathway in skin tumours. Nature 389: 876-881, 1997.

9. Watkins DN, Berman DM, Burkholder SG, et al: Hedgehog signalling within airway epithelial progenitors and in small-cell lung cancer. Nature 422: 313-317, 2003.

10. Berman DM, Karhadkar SS, Maitra A, et al: Widespread requirement for Hedgehog ligand stimulation in growth of digestive tract tumors. Nature 425: 846-851, 2003.

11. Thayer SP, Di Magliano MP, Heiser PW, et al: Hedgehog is an early and late mediator of pancreatic cancer tumorigenesis. Nature 425: 851-856, 2003

12. Karhadkar SS, Bova GS, Abdallah N, et al: Hedgehog signalling in prostate regeneration, neoplasia and metastasis. Nature 431 : 707-712, 2004

13. Katoh $\mathrm{Y}$ and Katoh $\mathrm{M}$ : Hedgehog signaling in gastric cancer. Cancer Biol Ther 4: 1050-1054, 2005.

14. Lindemann RK: Stroma-initiated Hedgehog signaling takes center stage in B-cell lymphoma. Cancer Res 68: 961-964, 2008.

15. Chamoun Z, Mann RK, Nellen D, et al: Skinny hedgehog, an acyltransferase required for palmitoylation and activity of the hedgehog signal. Science 293: 2080-2084, 2001.

16. Breitling R: Greased hedgehogs: new links between hedgehog signaling and cholesterol metabolism. BioEssays 29: 1085-1094, 2007.

17. Burke R, Nellen D, Bellotto M, et al: Dispatched, a novel sterolsensing domain protein dedicated to the release of cholesterolmodified hedgehog from signaling cells. Cell 99: 803-815, 1999.

18. Tenzen T, Allen BL, Cole F, et al: The cell surface membrane proteins Cdo and Boc are components and targets of the Hedgehog signaling pathway and feedback network in mice. Dev Cell 10: 647-656, 2006.

19. Allen BL, Tenzen T and McMahon AP: The Hedgehog-binding proteins Gas 1 and Cdo cooperate to positively regulate Shh signaling during mouse development. Genes Dev 21: 1244-1257, 2007.

20. Chuang PT and McMahon AP: Vertebrate Hedgehog signalling modulated by induction of a Hedgehog-binding protein. Nature 397: 617-621, 1999.

21. Van den Heuvel $\mathbf{M}$ and Ingham PW: Smoothened encodes a receptor-like serpentine protein required for hedgehog signalling. Nature 382: 547-551, 1996.

22. Gallet A and Therond PP: Temporal modulation of the Hedgehog morphogen gradient by a Patched-dependent targeting to lysosomal compartment. Dev Biol 277: 51-62, 2005.

23. Varjosalo M, Björklund M, Cheng F, et al: Application of active and kinase-deficient kinome collection for identification of kinases regulating hedgehog signaling. Cell 133: 537-548, 2008.

24. Taylor MD, Liu L, Raffel C, et al: Mutations in SUFU predispose to medulloblastoma. Nat Genet 31: 306-310, 2002.

25. Kinzler KW, Bigner SH, Bigner DD, et al: Identification of an amplified, highly expressed gene in a human glioma. Science 236: 70-73, 1987 .
26. Ruiz i Altaba A, Mas C and Stecca B: The Gli code: an information nexus regulating cell fate, stemness and cancer. Trends Cell Biol 17: 438-447, 2007.

27. Bhatia N, Thiyagarajan S, Elcheva I, et al: Gli2 is targeted for ubiquitination and degradation by $\beta$-TrCP ubiquitin ligase. $\mathrm{J}$ Biol Chem 281: 19320-19326, 2006.

28. Tempé D, Casas M, Karaz S, et al: Multisite protein kinase A and glycogen synthase kinase $3 B$ phosphorylation leads to Gli3 ubiquitination by SCF BTrCP. Mol Cell Biol 26: 4316-4326, 2006.

29. Hallikas O, Palin K, Sinjushina N, et al: Genome-wide prediction of mammalian enhancers based on analysis of transcriptionfactor binding affinity. Cell 124: 47-59, 2006

30. Kasper M, Schnidar H, Neill GW, et al: Selective modulation of Hedgehog/GLI target gene expression by EGF signaling in human keratinocytes. Mol Cell Biol 26: 6283-6299, 2006.

31. Katoh Y and Katoh M: WNT antagonist, SFRP1, is Hedgehog signaling target. Int J Mol Med 17: 171-175, 2006.

32. Katoh Y and Katoh M: Conserved POU-binding site linked to SP1-binding site within FZD5 promoter: transcriptional mechanisms of FZD5 in undifferentiated human ES cells, fetal liver/spleen, adult colon, pancreatic islet, and diffuse-type gastric cancer. Int J Oncol 30: 751-755, 2007.

33. Katoh $\mathbf{M}$ and Katoh $\mathbf{M}$ : Notch ligand, JAG1, is evolutionarily conserved target of canonical WNT signaling pathway in progenitor cells. Int J Mol Med 17: 681-685, 2006.

34. Katoh $\mathrm{M}$ and Katoh M: Conserved POU/OCT- and GATAbinding sites in 5'-flanking promoter region of mammalian WNT8B orthologs. Int J Oncol 30: 1273-1277, 2007.

35. Katoh Y and Katoh M: Comparative integromics on JMJD2A, JMJD2B and JMJD2C: preferential expression of JMJD2C in undifferentiated ES cells. Int J Mol Med 20: 269-273, 2007

36. Katoh $\mathrm{M}$ and Katoh $\mathrm{M}$ : Integrative genomic analyses on HES/HEY family: Notch-independent HES1, HES3 transcription in undifferentiated ES cells, and Notch-dependent HES1, HES5, $H E Y 1, H E Y 2, H E Y L$ transcription in fetal tissues, adult tissues, or cancer. Int J Oncol 31: 461-466, 2007.

37. Dennler S, André J, Alexaki I, et al: Induction of Sonic hedgehog mediators by TGF-3: Smad3-dependent activation of Gli2 and Gli1 expression in vitro and in vivo. Cancer Res 67: 6981-6986, 2007.

38. Katoh M and Katoh M: Crosstalk of WNT and FGF signaling pathways at GSK3ß to regulate $\beta$-catenin and SNAIL signaling cascades. Cancer Biol Ther 5: 1059-1064, 2006.

39. Katoh M: Networking of WNT, FGF, Notch, BMP, and Hedgehog signaling pathways during carcinogenesis. Stem Cell Rev 3: 30-38, 2007

40. Bailey J, Singh PK and Hollingsworth MA: Cancer metastasis facilitated by developmental pathways: Sonic hedgehog, Notch, and bone morphogenetic proteins. J Cell Biochem 102: 829-839, 2007.

41. Katoh M: Dysregulation of stem cell signaling network due to germline mutation, SNP, Helicobacter pylori infection, epigenetic change, and genetic alteration in gastric cancer. Cancer Biol Ther 6: 832-839, 2007.

42. Wei CL, Wu Q, Vega VB, et al: A global map of p53 transcription-factor binding sites in the human genome. Cell 124: 207-219, 2006.

43. Lefort K, Mandinova A, Ostano P, et al: NOTCH1 is a p53 target gene involved in human keratinocyte tumor suppression through negative regulation of ROCK $1 / 2$ and MRCK $\alpha$ kinases. Genes Dev 21: 562-577, 2007

44. Artavanis-Tsakonas S, Rand MD and Lake RJ: Notch signaling. Science 284: 770-776, 1999.

45. Radtke F and Raj K: The role of Notch in tumorigenesis. Nat Rev Cancer 3: 765-767, 2003.

46. Katoh $\mathrm{M}$ and Katoh M: Notch signaling in gastrointestinal tract. Int J Oncol 30: 247-251, 2007.

47. Katoh M, Hirai M, Sugimura T and Terada M: Cloning and characterization of MST, a novel serine/threonine kinase with SH3 domain. Oncogene 10: 1447-1451, 1995.

48. Riobó NA, Lu K, Ai X, Haines GM and Emerson CP Jr: PI3K and Akt are essential for Sonic Hedgehog signaling. Proc Natl Acad Sci USA 103: 4505-4510, 2006.

49. Smeenk L, van Heeringen SJ, Koeppel M, et al: Characterization of genome-wide p53-binding sites upon stress response. Nucleic Acids Res (In press).

50. Shmueli A and Oren M: Mdm2: p53's lifesaver? Mol Cell 25: 794-796, 2007. 
51. Gwack Y, Sharma S, Nardone J, et al: A genome-wide Drosophila RNAi screen identifies DYRK-family kinases as regulators of NFAT. Nature 441: 646-650, 2006.

52. Miller CT, Aggarwal S, Lin TK, et al: Amplification and overexpression of the dual-specificity tyrosine-(Y)-phosphorylation regulated kinase 2 (DYRK2) gene in esophageal and lung adenocarcinomas. Cancer Res 63: 4136-4143, 2003.

53. Gorringe KL, Boussioutas A, Bowtell DD, et al: Novel regions of chromosomal amplification at $6 \mathrm{p} 21,5 \mathrm{p} 13$, and $12 \mathrm{q} 14$ in gastric cancer identified by array comparative genomic hybridization. Genes Chromosomes Cancer 42: 247-259, 2005.

54. Momand J, Zambetti GP, Olson DC, George D and Levine AJ: The $M d m-2$ oncogene product forms a complex with the p53 protein and inhibits p53-mediated transactivation. Cell 69: $1237-1245,1992$.

55. Oliner JD, Kinzler KW, Meltzer PS, George DL and Vogelstein B: Amplification of a gene encoding a p53-associated protein in human sarcomas. Nature 358: 80-83, 1992.

56. Shvarts A, Steegenga WT, Riteco N, et al: MDMX: a novel p53-binding protein with some functional properties of MDM2. EMBO J 15: 5349-5357, 1996.
57. Marine JC, Dyer MA and Jochemsen AG: MDMX: from bench to bedside. J Cell Sci 120: 371-378, 2007.

58. Günther T, Schneider-Stock R, Häckel C, et al: $M d m 2$ gene amplification in gastric cancer correlation with expression of Mdm2 protein and p53 alterations. Mod Pathol 13: 621-626, 2000.

59. Riemenschneider MJ, Büschges R, Wolter M, et al: Amplification and overexpression of the MDM4 (MDMX) gene from 1q32 in a subset of malignant gliomas without TP53 mutation or MDM2 amplification. Cancer Res 59: 6091-6096, 1999.

60. Danovi D, Meulmeester E, Pasini D, et al: Amplification of $M D M X$ (or MDM4) directly contributes to tumor formation by inhibiting p53 tumor suppressor activity. Mol Cell Biol 24: 5835-5843, 2004

61. Laurie NA, Donovan SL, Shih CS, et al: Inactivation of the p53 pathway in retinoblastoma. Nature 444: 61-66, 2006.

62. Mayo LD and Donner DB: A PI3K/Akt pathway promotes translocation of Mdm2 from the cytoplasm to the nucleus. Proc Natl Acad Sci USA 98: 11598-11603,2001. 